\section{Associação da enrofloxacina à doxiciclina no tratamento do granuloma lepróide canino: relato de caso}

\author{
Enrofloxacin pool doxycycline treatment of canine granuloma \\ leproid: case report
}

Flávia Clare Goulart Carvalho*, Tábata Massessine Rosas², Marília Alves Machado³, Natália Lôres Lopes', Fabricia Hallack Loures ${ }^{4}$, Lissandro Gonçalves Conceição ${ }^{4} \&$ Julio Israel Fernandes ${ }^{5}$

'Médicos veterinários, MSc. Programa de Pós-graduação em Medicina Veterinária - PPGMV, Universidade Federal Rural do Rio de Janeiro - UFRRJ, Seropédica, RJ, Brasil

${ }^{2}$ Médica veterinária. Universidade Severino Sombra - USS, Vassouras, RJ, Brasil

${ }^{3}$ Médica veterinária. Programa de Pós-graduação em Medicina Veterinária - PPGMV, Universidade Federal Rural do Rio de Janeiro - UFRRJ, Seropédica, RJ, Brasil

${ }^{4}$ Médicos veterinários, DSc. Universidade Federal de Viçosa - UFV, Viçosa, MG, Brasil

${ }^{5}$ Médico veterinário, DSc. Departamento de Medicina e Cirurgia Veterinária, Universidade Federal Rural do Rio de Janeiro UFRRJ, Seropédica, RJ, Brasil

\section{Resumo}

Relata-se um caso de granuloma lepróide em um canino da raça Boxer proveniente da região Sul Fluminense, Estado do Rio de Janeiro, residente em sítio, apresentando lesões nodulares ulceradas, alopécicas, com exsudato serosanguinolento não doloroso e não pruriginoso, localizado em ambas as pinas e na coxa esquerda. O diagnóstico foi realizado a partir do exame citopatológico que revelou neutrófilos, plasmócitos, macrófagos, células gigantes multinucleadas, intensa atividade fagocitária e estruturas lineares coradas negativamente. O exame histopatológico evidenciou inflamação piogranulomatosa e com coloração especial de Ziehl-Neelsen que se mostrou positiva para BAAR. O protocolo terapêutico instituído foi a associação de enrofloxacina $10 \mathrm{mg} / \mathrm{kg}$ a cada 24 horas com doxiciclina $10 \mathrm{mg} / \mathrm{kg}$ a cada 24 horas, durante três meses. Foi observada cura clínica, sem recidiva e sem efeitos adversos ao tratamento.

Palavras-chave: micobacteriose, leproide, diagnóstico, tratamento.

\section{Abstract}

It is reported a case of canine leproid granuloma in a Boxer at South Fluminense region, in Rio de Janeiro State. Clinical signs included nodular ulcerated lesions, alopecia, and serosanguinous exudate with no complaint of pain or pruritus. The diagnosis was based on the cytopathological and histopathological examination, which revealed intense pyogranulomatous inflammation along with negative bacilli images or acid-fast bacilli, respectively. The treatment protocol was established with enrofloxacin $10 \mathrm{mg} / \mathrm{kg}$ once a day associated with doxycycline 10mg / kg once a day for 3 months with clinical cure without recurrence, and no side effects.

Keywords: mycobacteriosis, leproid, diagnosis, treatment.

\section{Introdução}

O granuloma lepróide canino (GLC) é causado por uma micobactéria que ainda não foi tipificada e que se relaciona filogeneticamente com Mycobacterium simiae, Mycobacterium tilburgiie, Mycobacterium genavense (Foley et al., 2002) e no Brasil com a Mycobacterium Murphy (Conceição et al., 2011).

Oprimeiro caso identificado no Brasil foi no Estado de São Paulo, no ano de1990 (Larsson et al., 1994). E hoje já existem relatos nos Estados de Goiás, Paraná, Minas Gerais, Distrito Federal, Rio de Janeiro, Bahia (Larsson \& Lucas, 2016) e Pará (Almeida et al., 2013).

Estudos descrevem a raça Boxer e mestiços da mesma como os mais propensos a desenvolverem a doença (Larsson \& Lucas, 2016). Acredita-se que a inoculação da micobactéria nos animais ocorra a partir do repasto sanguíneo de insetos (Malik et al., 1998). As lesões ocorrem geralmente nas pinas e cabeça, por serem regiões anatômicas mais susceptíveis a picadas de inseto por possuírem uma maior vascularização superficial e uma densidade pilosa menor. Entretanto, outras formas de
Como citar: Carvalho, F.C. G, Rosas, T. M., Machado, M. A., Lopes, N. L., Loures, F. H., Conceição, L. G., \& Fernandes, J. I. (2017). Associação da enrofloxacina à doxiciclina no tratamento do granuloma lepróide canino: relato de caso. Brazilian Journal of Veterinary Medicine, 39(3), 203-207. doi: 10.29374/2527-2179.bjvm008217

Fonte de financiamento: Recurso próprio.

Conflito de interesses: Os autores declaram não haver conflito de interesses que precisam ser informados.

Recebido: Abril 17, 2017

Aceito: Agosto 12, 2017.

Oestudo foi realizado na Universidade Severino Sombra - USS, Vassouras, RJ, Brasil.

\section{Correspondência}

Flávia Clare Goulart Carvalho

Universidade Federal Rural do Rio de Janeiro - UFRRJ

Rodovia BR 465, Km 07

CEP 23890-000 - Seropédica (RJ), Brasil

E-mail: flaviaclare@gmail.com
Copyright Carvalho et al. Este é um artigo publicado em acesso aberto (Open Access) sob a licença Creative Commons Attribution Non-Commercial, que permite uso, distribuição e reprodução em qualquer meio, sem restrições desde que sem fins comerciais e que o trabalho original seja corretamente citado. 
infecção através de material cirúrgico e solo contaminados também são descritas (Maliket al., 2006), bem como outros locais podem ser acometidos, como os membros (Foley et al., 2002).

As lesões se caracterizam inicialmente por pápulas e progridem para nódulos que posteriormente ulceram. Frequentemente, há o acometimento da derme e do tecido subcutâneo (Foley et al., 2002). Muitos casos são descritos como autolimitantes, apresentando regressão espontânea entre um e três meses após o seu aparecimento (Malik et al., 1998).

O diagnóstico clínico baseia-se nas lesões localizadas em locais de temperatura corpórea mais baixa, além do porte do animal, da raça e da pelagem curta (Larsson \& Maruyama, 2008). Porém, a confirmação do diagnóstico ocorre através do exame citopatológico ou histopatológico, onde observa-se infiltrado inflamatório piogranulomatoso com presença variável de bacilos álcool ácido resistentes na coloração de Ziehl-Neelsen (Malik et al., 2006; Almeida et al., 2013). Os diagnósticos diferenciais são granulomas por corpos estranhos, prototecose, actinomicose, esporotricose, pseudomicetoma (Larsson et al., 2006 ) e neoplasias (Almeida et al., 2013).

Para o tratamento pode ser utilizada a mono ou poliantibioticoterapia. A combinação de antibióticos como a claritromicina, doxiciclina e rifampicina podem ser utilizados (Larsson \& Maruyama, 2008). Protocolos monoterápicos com o uso de rifampicina 10 a 15mg/kg/SID/VO ou enrofloxacina $5 \mathrm{mg} / \mathrm{kg} / \mathrm{SID} / \mathrm{VO}$ em conjunto com terapia tópica a base de rifampicina apresentam resultados satisfatórios (Larsson \& Lucas, 2016). Objetivou-se descrever as características clínicas, o diagnóstico, o tratamento e a evolução encontrados em um caso de GLC atendido no município de Vassouras/RJ.

\section{Histórico}

Foi atendido em uma Clínica Veterinária, em Vassouras/RJ, um cão da raça Boxer, macho, inteiro, com sete anos de idade, com a queixa de lesões cutâneas nos pavilhões auriculares há 45 dias. O tratamento prévio com cefalexina 30mg/kg a cada 12 horas por 30 dias não resultou em melhora clínica, e nesse período, as lesões evoluíram para pior.

Não apresentou prurido ou apatia, porém notou-se hiporexia e perda peso. Ao exame físico o animal apresentava normotermia, mucosas normocoradas, hidratado, sem linfadenomegalia, ausculta cardiopulmonar e palpação abdominal normais. As lesões cutâneas caracterizavam-se por múltiplos nódulos alopécicos, ulcerados, eritematosos com um a quatro centímetros de diâmetro, encimados por discreto exsudato soro sanguinolento, não dolorosos, de consistência firme a fibroelástica, localizados na face convexa de ambos os pavilhões auriculares (Figura 1a, b) como também na região lateral da coxa direita.

Suspeitando-se inicialmente de granuloma lepróide canino e esporotricose foram coletados materiais para exames citológico e histopatológico de pele.

Para o exame citológico, as lâminas contendo material coletado por decalque e por punção aspirativa por agulha fina foram coradas pelo método Panótico Rápido e examinadas em microscopia ótica de luz. O exame revelou elevada celularidade, com predomínio de neutrófilos integros, plasmócitos, grande número de macrófagos com intensa atividade fagocitária, células gigantes multinucleadas, por vezes contendo estruturas baciliformes em imagem negativa (Figura 2). Foi realizada a excisão cirúrgica de dois nódulos da pina esquerda, acondicionados em formol a 10\% e encaminhados para a realização do histopatológico.

O exame histopatológico revelou em grande extensão dos fragmentos, intenso infiltrado inflamatório difuso, misto, piogranulomatoso e plasmocitário, formados pela coalescência de infiltrados nodulares menores, acompanhados de áreas de necrose, trechos de hemorragia, ectasia e congestão vascular (Figura 3). A coloração especial de Ziehl-Neelsen mostrou-se positiva para BAAR. Coloração especial de PAS foi negativa para estruturas fúngicas.

Com diagnóstico de micobacteriose e compatibilidade anatomo-clínica com GLC iniciou-se o tratamento via oral com enrofloxacina $10 \mathrm{mg} / \mathrm{kg}$ cada 24 horas associado à doxiciclina $10 \mathrm{mg} / \mathrm{kg}$ a cada 24 horas, omeprazol $1 \mathrm{mg} / \mathrm{kg} / \mathrm{a}$ cada 24 horas e rifampicina tópica duas vezes ao dia. Dois meses após o início do tratamento observou-se melhora na ordem de 80\% das lesões dermatológicas. Ao final do terceiro mês, o animal não mais apresentava lesões cutâneas, sem o relato de efeito colateral à medicação ou recidivas das lesões em ambas as orelhas (Figura 4a, b). 


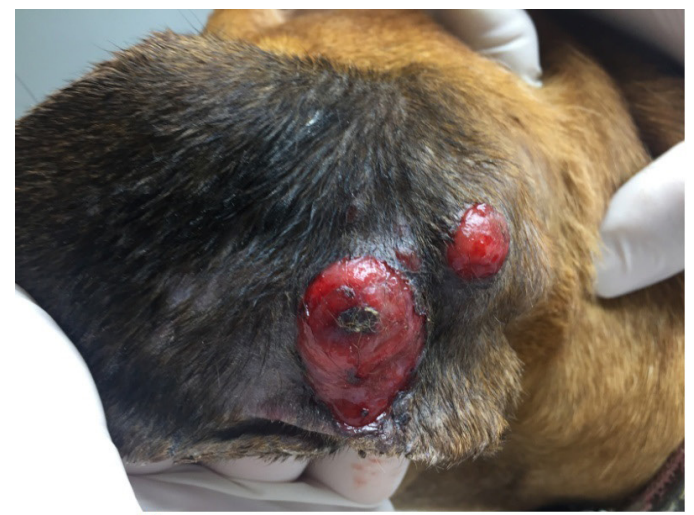

(a)

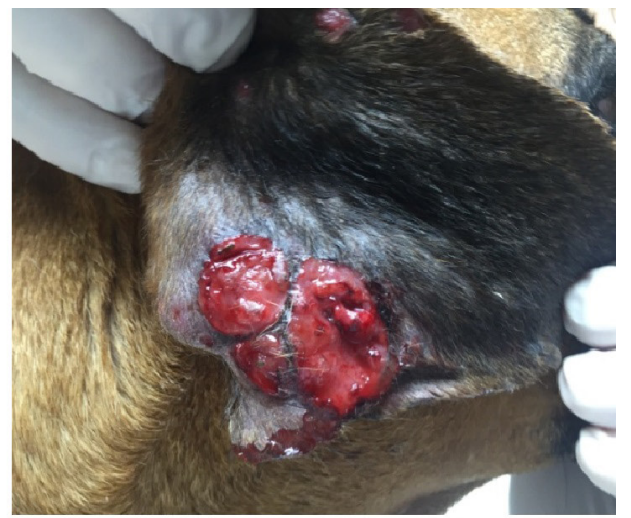

(b)

Figura 1. (a) Lesões nodulares ulceradas localizadas na face convexa do pavilhão auricular esquerdo em cão da raça boxer, sete anos de idade atendido em clínica veterinária em Vassouras diagnosticado com granuloma lepróide canino; (b) Lesões nodulares ulceradas, com perda tecidual, friáveis, na face convexa do pavilhão auricular direito em cão da raça boxer, sete anos de idade atendido em clínica veterinária em Vassouras diagnosticado com granuloma lepróide canino.

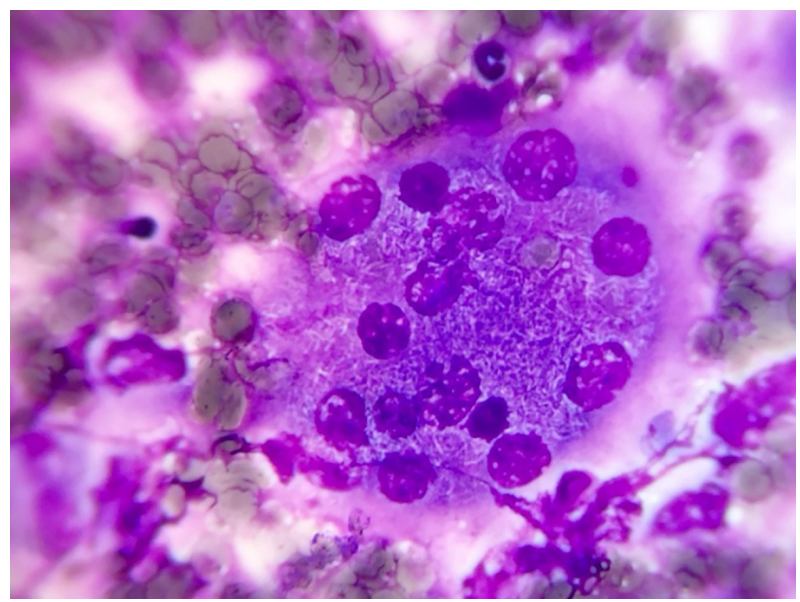

Figura 2. Exame citológico em canino raça boxer, sete anos de idade atendido em clínica veterinária em Vassouras diagnosticado com granuloma lepróide apresentando células gigantes multinucleadas com estruturas lineares não coradas (X1000).

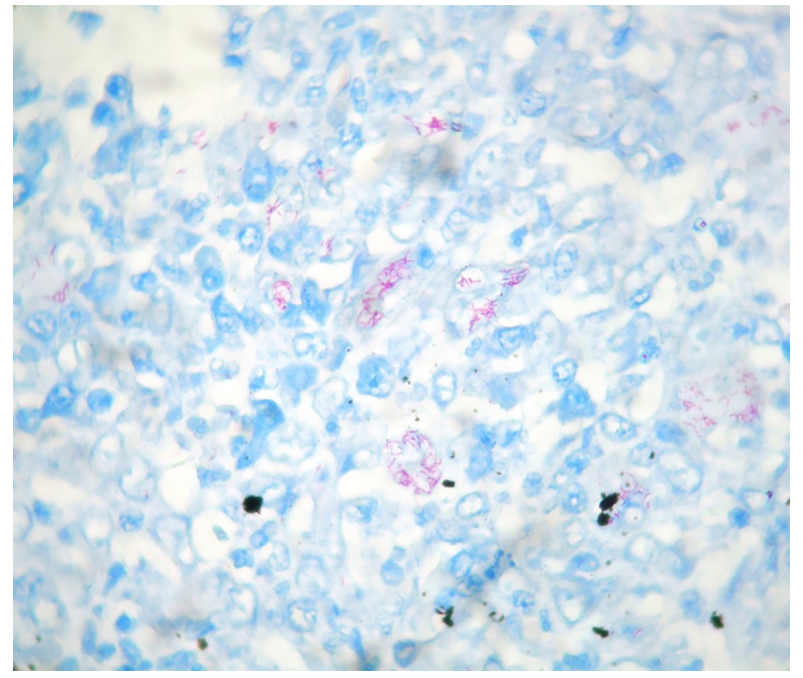

Figura 3. Exame histopatológico citológico em canino raça boxer, sete anos de idade atendido em clínica veterinária em Vassouras diagnosticado com granuloma lepróide, com coloração especial de Ziehl-Neelsen positiva para bacilos álcool ácido resistentes (X1000). 


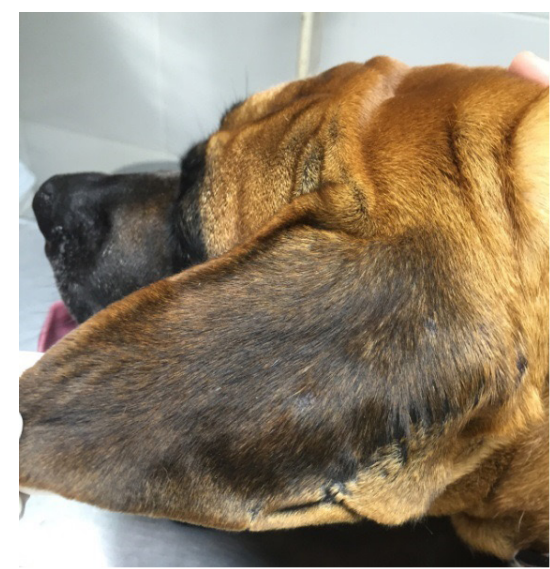

(a)

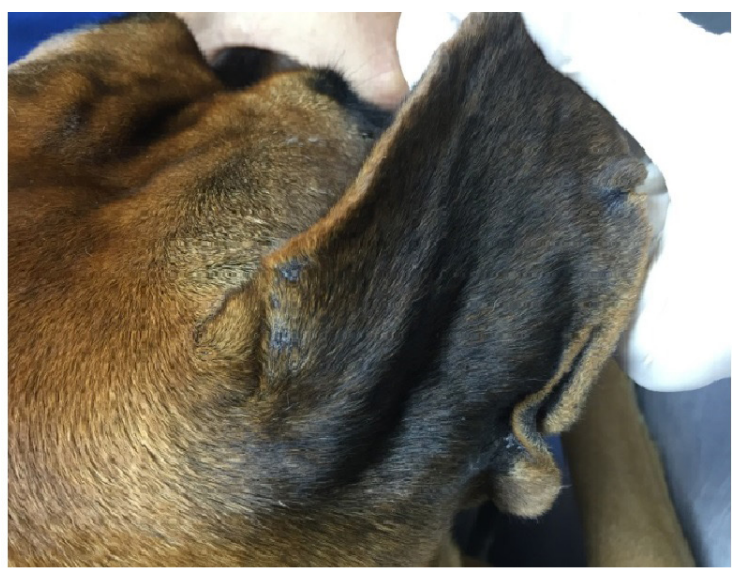

(b)

Figura 4. (a) e (b): Completa resolução clínica em revisão de 90 dias de um canino da da raça boxer, sete anos de idade atendido em clínica veterinária em Vassouras diagnosticado com granuloma lepróide canino após tratamento com associação de enrofloxacina com doxiciclina a cada 24 horas.

\section{Discussão}

A micobactéria que origina o GLC ainda não apresenta etiologia definida, mas correlaciona-se o repasto sanguíneo realizado por insetos como possível fonte de inoculação da mesma (Maliketal.,1998). O local onde o animal reside é de grande importância, visto que animais que possuem acesso à ambientes externos possuem um contato maior com insetos (Conceição et al., 2011).

A descrição da forma de inoculação das micobactérias favorece a ocorrência nas regiões de pina, sendo relatado na literatura como as mais acometidas (Malik et al., 2006), corroborando com os estudos de Almeida et al. (2013), que descreve as localizações das lesões como uni ou bilateral, o que pode ser observado no trabalho apresentado. A raça descrita no caso corrobora com Almeida et al. (2013) e Larsson \& Lucas (2016), que citam o Boxer como o mais propenso a desenvolver o GLC, não apenas por fatores genéticos, mas também em relação ao tipo de pelame curto, o que pode favorecer a inoculação por insetos. Conceição et al. (2011) notaram que cães com fenótipos parecidos com a raça Boxer não são afetados com a mesma intensidade; dessa forma, a predisposição da raça pode estar relacionada também a uma resposta imunológica deficiente e não apenas com suas características físicas; porém são necessários mais estudos.

As lesões apresentadas no relato acima estão de acordo com as descritas por Foley et al. (2002) onde nódulos ulcerados, não dolorosos e com ausência de prurido são característicos. Lesões nodulares, alopécicas e ulceradas em ambas as pinas também foram descritas por Almeida et al. (2013). Assim como descrito por Malik et al. (1998), não foi evidenciado o acometimento de outros órgãos no caso relatado. Já com relação ao relato de Larsson et al. (2006) observou-se alterações clínicas como oligodipsia, episódios de êmese e anorexia, sendo estas alterações incomuns em quadros de micobacteriose.

A esporotricose éuma doença fúngica e pode manifestar-se de forma semelhante e por isso, esta zoonose é considerada um importante diagnóstico diferencial (Scott et al., 1996) principalmente em áreas endêmicas, como o Estado do Rio de Janeiro.

Em estudos anteriores, o uso da enrofloxacina e da rifampicina tópica não obteve a cura clínica e apresentou efeitos adversos (Larsson \& Maruyama, 2008). O tratamento preconizado com a associação da enrofloxacina à doxiciclina e o uso da rifampicina tópica foi eficaz e sem efeitos adversos. Como descrito por Larsson et al. (2006) o uso de quinolonas são melhores escolhas de antibioticoterapia sistêmica, assim como a terapia tópica com o uso da rifampicina, apresentando ação micobactericida. O resultado obtido com o tratamento utilizado corrobora com os estudos de Malik et al. (2001), onde associações de enrofloxacina e doxiciclina são boas opções de terapia, mas estão disponíveis vários protocolos com diferentes respostas. Almeida et al. (2013), suspeitaram inicialmente, de quadro neoplásico; assim, optou-se pela retirada cirúrgica com ampla margem como opção de tratamento, o que difere do presente caso, onde, apesar das 
neoplasias estarem incluídas nos diagnósticos diferenciais, a terapia antibiótica sistêmica foi preconizada como primeira escolha.

\section{Conclusão}

A terapia com enrofloxacina associada à doxiciclina foi eficaz, com remissão completa das lesões em 90 dias e sem efeitos colaterais. Deve-se considerar o GLC como um diagnóstico diferencial na clínica, principalmente nas regiões endêmicas para esporotricose, além das neoplasias, sendo a citopatologia e a histopatologia métodos de diagnósticos conclusivos para GLC.

\section{Referências}

Almeida, M. B., Priebe, A. P. S., Fernandes, J. I., Yamasaki, E. M., \& França, T. N. (2013). Granuloma leproide canino na região amazônica - relato de caso. Arquivo Brasileiro de Medicina Veterinária e Zootecnia, 65(3), 645-648. http://dx.doi.org/10.1590/S0102-09352013000300004.

Conceição, L. G., Acha, L. M. R., Borges, A. S., Assis, F. G., Loures, F. H., \& Silva, F. F. (2011). Epidemiology, clinical signs, histopathology and molecular characterization of canine leproid granuloma: a retrospective study of cases from Brazil. Veterinary Dermatology, 22(3), 249-256. http://dx.doi.org/10.1111/j.1365-3164.2010.00934.x. PMid:21410568.

Foley, J. E., Borjesson, D., L Gross, T., Rand, C., Needham, M., \& Poland, A. (2002). Clinical, microscopic and molecular aspects of canine leproid granuloma in the United States. Veterinary Pathology, 39(2), 234-239. http://dx.doi.org/10.1354/vp.39-2-234. PMid:12009061.

Larsson, C. E., \& Lucas, R. (2016). Micobacterioses tegumentares. In S. Maruyama (Eds.), Tratado de medicina externa: dermatologia veterinária (1. ed., pp. 478-485). São Caetano do Sul: Interbook.

Larsson, C. E., \& Maruyama, S. (2008). Micobacterioses. Revista Clínica Veterinária, 72, 36-44.

Larsson, C. E., Delayte, E. H., Balda, A. C., Michalany, N. S., Pinheiro, S. R., Otsuka, M., \& Roxo, E. (2006). Dermatite micobacteriana atípica em gato: relato de caso. Arquivo Brasileiro de Medicina Veterinária e Zootecnia, 58(6), 1092-1098. http://dx.doi.org/10.1590/S0102-09352006000600018.

Larsson, C. E., Michalany, N., Pinheiro, S. R., Ledon, A. L. B. P., \& Vasconcellos, S. A. (1994). Mycobacteriosis in domestic dogs. Report of two cases in São Paulo. Revista da Faculdade de Medicina Veterinária e Zootecnia da Universidade de São Paulo, 31, 35-41.

Malik, R., Hughes, M. S., Martin, P., \& Wigney, D. (2006). Canine leproid granuloma syndrome (Canine leprosy). In C. E. Greene (Eds.), Infectious diseases of the dog and cat (pp. 480-482). St Louis: Saunders Elsevier.

Malik, R., Love, D. N., Wigney, D. I., \& Martin, P. (1998). Mycobacterial nodular granulomas affecting the subcutis and skin of dogs (canine leproid granuloma syndrome). Australian Veterinary Journal, 76(6), 403-407, 398. http://dx.doi.org/10.1111/j.1751-0813.1998.tb12388.x. PMid:9673764.

Malik, R., Martin, P., Wigney, D., Swan, D., Slatter, P. S., Cibilic, D., Allen, J., Mitchell, D. H., Chen, S. C., Hughes, M. S., \& Love, D. N. (2001). Treatment of canine leproid granuloma syndrome: preliminary findings in seven dogs. Australian Veterinary Journal, 79(1), 30-36. http://dx.doi.org/10.1111/j.1751-0813.2001.tb10635.x. PMid:11221566.

Scott, D. W., Miller, H. W., \& Griffin, C. E. (1996). Muller \& Kirk dermatologia dos pequenos animais (5. ed., 1130 p.). São Paulo: Manole. 